\title{
Effect of Hall Current on Unsteady Magneto Hydrodynamic Flow Past an Exponentially Accelerated Inclined Plate with Variable Temperature and Mass Diffusion
}

\author{
U. S. Rajput and Gaurav Kumar \\ Department of Mathematics and Astronomy \\ University of Lucknow, Lucknow 226007 U.P, India \\ *Corresponding author: rajputgauravlko@gmail.com
}

Article history

Received: 22 September 2016

Received in revised form: 29 July 2018

Accepted: 2 August 2018

Published on line: 1 December 2018

\begin{abstract}
The present study is carried out to examine the effect of Hall current on unsteady flow of a viscous, incompressible and electrically conducting fluid past an exponentially accelerated inclined plate with variable wall temperature and mass diffusion in the presence of transversely applied uniform magnetic field. The plate temperature and the concentration level near the plate increase linearly with time. The governing equations involved in the present analysis are solved by the Laplace-transform technique. The velocity profile is discussed with the help of graphs drawn for different parameters like thermal Grashof number, mass Grashof number, Prandtl number, Hall current parameter, acceleration parameter, the magnetic field parameter and Schmidt number, and the numerical values of skin-friction have been tabulated. It is observed that the flow pattern is affected significantly with plate acceleration, Hall current. The importance of the problem can be seen in cooling of electronic components of a nuclear reactor, bed thermal storage and heat sink in the turbine blades.
\end{abstract}

Keywords Magneto hydrodynamic flow; inclined plate; variable temperature; mass diffusion; Hall current.

Mathematics Subject Classification 76W05, 76D05.

\section{Introduction}

The MHD flow problems play important roles in different area of science and technology, like biological science, petroleum engineering, chemical engineering, mechanical engineering, biomechanics, irrigation engineering and aerospace technology. The influence of magnetic field on viscous, incompressible and electrically conducting fluid is of great importance in many applications such as magnetic material processing, glass manufacturing control processes and purification of crude oil. The response of laminar skin friction and heat transfer to fluctuations in the stream velocity was studied by Lighthill [1]. Rajput and Kumar [11] have analyzed MHD 
flow past an impulsively started vertical plate with variable temperature and mass diffusion. Free convection flow past an exponentially accelerated vertical plate was considered by Singh and Kumar[4]. Mass transfer effects on the flow past an exponentially accelerated vertical plate with constant heat flux was studied by Basant et al. [5]. Muthucumaraswamy et al. [9] have considered heat transfer effect on flow past an exponentially accelerated vertical plate with variable temperature. Muthucumaraswamy et al. [10] have studied mass transfer effects on exponentially accelerated isothermal vertical plate. Hall current effect on MHD flow is also significant in many cases. Some such problems already studied are mention here. Katagiri [2] has investigated the effect of Hall current on the magneto hydrodynamic boundary layer flow past a semi-infinite fast plate. Pop [5] has considered the effect of Hall currents on hydromagnetic flow near an accelerated plate. Hall effect on magnetohydrodynamic boundary layer flow over a continuous moving flat plate was investigated by Pop and Watanabe [6]. Attia [7] has analyzed the effect of variable properties on the unsteady Hartmann flow with heat transfer considering the Hall effect. Further, Attia and Sayed [8] have studied the Hall effect on unsteady MHD couette flow with heat transfer of a bingham fluid with suction and injection. Thamizhsudar and Pandurangan[12] have considered combined effects of radiation and Hall current on MHD flow past an exponentially accelerated vertical plate in the presence of rotation. Srinivas and Naikoti [13] has analyzed Hall effect on unsteady MHD free convection flow over a stretching sheet with variable viscosity and viscous dissipation. We are considering the unsteady MHD flow past an exponentially accelerated inclined plate with variable temperature and mass diffusion in the presence of Hall current. The results are shown with the help of graphs and table.

\section{Mathematical Analysis}

The geometrical model of the problem is shown in Figure-1

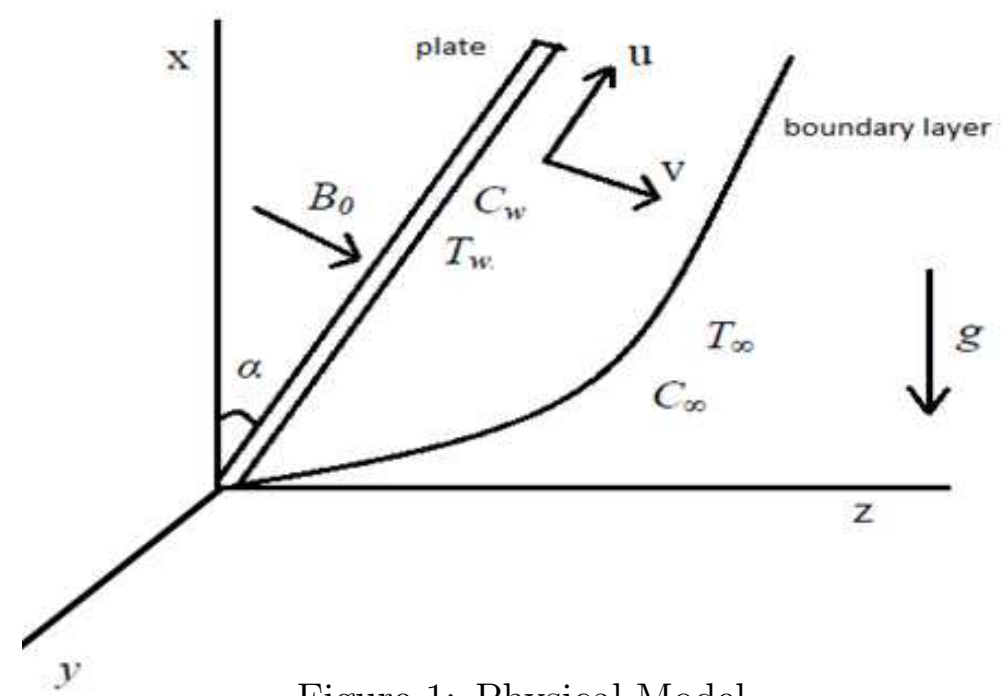

Figure 1: Physical Model

Consider an unsteady flow of a viscous, incompressible, electrically conducting fluid past an impulsively started non-conducting inclined flat plate. The $\mathrm{x}$ axis is taken along the vertical 
plane and $\mathrm{z}$ axis is normal to it. Thus the $\mathrm{z}$ axis lies in the horizontal plane. The plate is inclined at angle $\alpha$ from vertical. A transverse magnetic field $B_{0}$ of uniform strength is applied on the flow. Initially it has been considered that the plate as well as the fluid is at the same temperature $T_{\infty}$. The species concentration in the fluid is taken as $C_{\infty}$. At time $t>0$, the plate starts exponentially accelerating in its own plane with velocity $u=u_{0} e^{b t}$, and temperature of the plate is raised to $T_{w}$. The concentration $C$ near the plate is raised linearly with respect to time.

The flow model is as follows:

$$
\begin{aligned}
& \frac{\partial u}{\partial t}=v \frac{\partial^{2} u}{\partial z^{2}}+g \beta \cos \alpha\left(T-T_{\infty}\right)+g \beta^{*} \cos \alpha\left(C-C_{\infty}\right)-\frac{\sigma B_{0}^{2}(u+m v)}{\rho\left(1+m^{2}\right)}, \\
& \frac{\partial v}{\partial t}=v \frac{\partial^{2} v}{\partial z^{2}}+\frac{\sigma B_{0}^{2}(m u-v)}{\rho\left(1+m^{2}\right)}, \\
& \frac{\partial C}{\partial t}=D \frac{\partial^{2} C}{\partial z^{2}} \\
& \rho C_{p} \frac{\partial T}{\partial t}=k \frac{\partial^{2} T}{\partial z^{2}} .
\end{aligned}
$$

The initial and boundary conditions are

$$
\begin{aligned}
& t \leq 0: u=0, v=0, T=T_{\infty}, C=C_{\infty}, \text { for all } z, \\
& \left.\begin{array}{l}
t>0: u=u_{0} e^{b t}, v=0, T=T_{\infty}+\left(T_{w}-T_{\infty}\right) \frac{u_{0}^{2} t}{v}, C=C_{\infty}+\left(C_{w}-C_{\infty}\right) \frac{u_{0}^{2} t}{v}, z=0, \\
u \rightarrow 0, v \rightarrow 0, T \rightarrow T_{\infty}, C \rightarrow C_{\infty} \text { as } z \rightarrow \infty
\end{array}\right\}
\end{aligned}
$$

The following non-dimensional quantities are introduced to transform equations (1), (2), (3) and (4) into dimensionless form:

$$
\left.\begin{array}{l}
\bar{z}=\frac{z u_{0}}{v}, \bar{u}=\frac{u}{u_{0}}, \bar{v}=\frac{v}{u_{0}}, \theta=\frac{\left(T-T_{\infty}\right)}{\left(T_{w}-T_{\infty}\right)}, S_{c}=\frac{v}{D}, \mu=\rho v, P_{r}=\frac{\mu c_{p}}{k}, G_{r}=\frac{g \beta v\left(T_{w}-T_{\infty}\right)}{u_{0}^{3}}, \\
M=\frac{\sigma B_{0}^{2} v}{\rho u_{0}^{2}}, G_{m}=\frac{g \beta^{*} v\left(C_{w}-C_{\infty}\right)}{u_{0}^{3}}, \bar{C}=\frac{\left(C-C_{\infty}\right)}{\left(C_{w}-C_{\infty}\right)}, \bar{b}=\frac{b v}{u_{0}^{2}}, \bar{t}=\frac{t u_{0}^{2}}{v}, m=\omega_{e} \tau_{e} .
\end{array}\right\}
$$

Thus the model becomes

$$
\begin{aligned}
& \frac{\partial \bar{u}}{\partial \bar{t}}=\frac{\partial^{2} \bar{u}}{\partial \bar{z}^{2}}+G_{r} \cos \alpha \theta+G_{m} \cos \alpha \bar{C}-\frac{M(\bar{u}+m \bar{v})}{\left(1+m^{2}\right)}, \\
& \frac{\partial \bar{v}}{\partial \bar{t}}=\frac{\partial^{2} \bar{u}}{\partial \bar{z}^{2}}+\frac{M(m \bar{u}-\bar{v})}{\left(1+m^{2}\right)}, \\
& \frac{\partial \bar{C}}{\partial \bar{t}}=\frac{1}{S_{c}} \frac{\partial^{2} \bar{C}}{\partial \bar{z}^{2}} \\
& \frac{\partial \theta}{\partial \bar{t}}=\frac{1}{P_{r}} \frac{\partial^{2} \theta}{\partial \bar{z}^{2}} .
\end{aligned}
$$

The corresponding boundary conditions (5) become:

$$
\left.\begin{array}{l}
\bar{t} \leq 0: \bar{u}=0, \bar{v}=0, \theta=0, \bar{C}=0, \text { for all } \bar{z}, \\
\bar{t}>0: \bar{u}=e^{\bar{b} \bar{t}}, \bar{v}=0, \theta=\bar{t}, \bar{C}=\bar{t}, \text { at }, \bar{z}=0, \\
\bar{u} \rightarrow 0, \bar{v} \rightarrow 0, \theta \rightarrow 0, \bar{C} \rightarrow 0, \text { as } \bar{z} \rightarrow \infty .
\end{array}\right\}
$$


Dropping bars in the above equations, we get

$$
\begin{aligned}
\frac{\partial u}{\partial t} & =\frac{\partial^{2} u}{\partial z^{2}}+G_{r} \operatorname{Cos} \alpha \theta+G_{m} \operatorname{Cos} \alpha C-\frac{M(u+m v)}{\left(1+m^{2}\right)}, \\
\frac{\partial v}{\partial t} & =\frac{\partial^{2} v}{\partial z^{2}}+\frac{M(m u-v)}{\left(1+m^{2}\right)}, \\
\frac{\partial C}{\partial t} & =\frac{1}{S_{c}} \frac{\partial^{2} C}{\partial z^{2}} \\
\frac{\partial \theta}{\partial t} & =\frac{1}{P_{r}} \frac{\partial^{2} \theta}{\partial z^{2}} .
\end{aligned}
$$

The boundary conditions are

$$
\left.\begin{array}{l}
t \leq 0: u=0, v=0, \theta=0, C=0, \text { for all } z, \\
t>0: u=e^{b t}, v=0, \theta=t, C=t \text { at } z=0, \\
\mathrm{u} \rightarrow 0, \mathrm{v} \rightarrow 0, \theta \rightarrow 0, \mathrm{C} \rightarrow 0, \text { as } z \rightarrow \infty .
\end{array}\right\}
$$

Writing the equations (12) and (13) in combined form (using $q=u+i v$ )

$$
\begin{aligned}
& \frac{\partial q}{\partial t}=\frac{\partial^{2} q}{\partial z^{2}}+G_{r} \cos \alpha \theta+G_{m} \cos \alpha C-q a, \\
& \frac{\partial C}{\partial t}=\frac{1}{S_{c}} \frac{\partial^{2} C}{\partial z^{2}} \\
& \frac{\partial \theta}{\partial t}=\frac{1}{P_{r}} \frac{\partial^{2} \theta}{\partial z^{2}} .
\end{aligned}
$$

The boundary conditions become:

$$
\left.\begin{array}{l}
t \leq 0: q=0, \theta=0, C=0, \text { for all } z, \\
t>0: q=e^{b t}, \theta=t, C=t, \text { at } z=0, \\
q \rightarrow 0, \theta \rightarrow 0, C \rightarrow 0, \text { as } z \rightarrow \infty .
\end{array}\right\}
$$

The dimensionless governing equations (17) to (19), subject to the boundary conditions (20), are solved by the usual Laplace - transform technique. The solution obtained is as follows:

$$
\begin{aligned}
C= & t\left\{\left(1+\frac{z^{2} S_{c}}{2 t}\right) \operatorname{erfc}\left[\frac{\sqrt{S_{c}}}{2 \sqrt{t}}\right]-\frac{z \sqrt{S_{c}}}{\sqrt{\pi \sqrt{t}}} e^{\left.-\frac{z^{2}}{4 t} S_{c}\right\}}\right. \\
\theta= & t\left\{\left(1+\frac{z^{2} P_{r}}{2 t}\right) \operatorname{erf} c\left[\frac{\sqrt{P_{r}}}{2 \sqrt{t}}\right]-\frac{z \sqrt{P_{r}}}{\sqrt{\pi \sqrt{t}}} e^{-\frac{z^{2}}{4 t}} P_{r}\right\}, \\
q= & \frac{1}{2} e^{b t-\sqrt{a+b} z} A_{15}+\frac{\cos \alpha}{4 a^{2} \sqrt{\pi}}\left[\sqrt{\pi} G_{r}\left\{-A_{9} z+\sqrt{a} e^{-\sqrt{a} z} A_{2} z+\frac{1}{2 A_{13} A_{3}}+2 e^{-\sqrt{a} z} A_{1} P_{r}+2 A_{13} A_{3} P_{r}\right\}\right. \\
& -G_{r} P_{r}\left\{-a A_{10} z+\frac{1}{\sqrt{P_{r}}} A_{13} \sqrt{\pi} A_{4}+\frac{2 \sqrt{\pi} A_{11}}{\sqrt{P_{r}}}-\frac{2 a \sqrt{\pi} t A_{11}}{\sqrt{P_{r}}}+\frac{1}{A_{13} \sqrt{\pi} A_{8} \sqrt{P_{r}}}-2 \sqrt{\pi P_{r}} A_{11}\right\} \\
& -\sqrt{\pi} G_{m}\left\{A_{9} z-\sqrt{a} e^{-\sqrt{a} z} A_{2} z-2 e^{-\sqrt{a} z} A_{1} S_{c}+2 A_{14} A_{5} S_{c}\right\} \\
& \left.+\sqrt{S_{c}} G_{m}\left\{-a A_{16} z+\frac{1}{\sqrt{\pi S_{c}} A_{14} A_{7}}+\frac{2 \sqrt{\pi} A_{12}}{\sqrt{S_{c}}}+\frac{1}{A_{14} \sqrt{\pi S_{c}} A_{6}}-2 A_{12} \sqrt{\pi S c}\right\}\right] .
\end{aligned}
$$


The expressions for the symbols involved in the above solutions are given in the appendix.

\section{Skin Friction}

The dimensionless skin friction at the plate is

$$
\left(\frac{d q}{d z}\right)_{z=0}=\tau_{x}+i \tau_{y} .
$$

The numerical values of $\tau_{x}$ and $\tau_{y}$, for different parameters are given in Table- 1 .

\section{Result and Discussions}

The velocity profile for different parameters like, thermal Grashof number $(G r)$, mass Grashof number $(G m)$, magnetic field parameter $(M)$, Hall parameter $(m)$, acceleration parameter $(b)$, Prandtl number $(P r)$ and time $(t)$ is shown in figures 1.1 to 2.8. It is observed from figures 1.1 and 2.1 that the primary and secondary velocities of fluid decrease when the angle of inclination of plat $(\alpha)$ is increased. It is observed from figure 1.2 and 2.2 , when the mass Grashof number $G m$ is increased then the velocities are increased. From figures 1.3 and 2.3 it is deduced that when thermal Grashof number $G r$ is increased then the velocities get increased. If Hall current parameter $m$ is increased then the primary velocity increased and secondary velocity decreased (figures 1.4 and 2.4). It is observed from figures 1.5 and 2.5 that the effect of increasing values of the parameter $M$ results in decreasing $\mathrm{u}$ and increasing $v$. It is deduced that when acceleration parameter is increased then the velocities are increased (figures 1.6 and 2.6). Further, it is observed that velocities decrease when Prandtl number is increased (figures 1.7 and 2.7). When the Schmidt number increased then the velocities get decreased (figures 1.8 and 2.8). Further, from figures 1.9 and 2.9 it is observed that velocities increase with time.

Skin friction is given in Table 1 . The value of $\tau_{\mathrm{x}}$ increases with the increase in thermal Grashof number, mass Grashof Number, Hall current parameter and time, and it decreases with the angle of inclination of plate, the magnetic field parameter, acceleration parameter, Prandtl number and Schmidt number. Similar effect is observed with $\tau_{\mathbf{x}}$, except magnetic field parameter, acceleration parameter and Hall parameter, in which case $\tau_{\mathrm{y}}$ increases with magnetic field parameter and acceleration parameter, and decreases with Hall parameter.

\section{Conclusion}

The conclusions of the study are as follows:

- Primary velocity increases with the increase in thermal Grashof number, mass Grashof number, Hall current parameter, acceleration parameter and time.

- Primary velocity decreases with angle of inclination of plate, the magnetic field, Prandtl number and Schmidt number.

- Secondary velocity increases with the increase in thermal Grashof number, mass Grashof number, the magnetic field, acceleration parameter and time. 
- Secondary velocity decreases with the angle of inclination of plate, Hall current parameter, Prandtl number and Schmidt number.

- $\tau_{\mathrm{x}}$ increases with the increase in $G r, G m, m$ and $t$, and it decreases with $\alpha, M, b, \operatorname{Pr}$ and Sc.

- $\tau_{\mathrm{y}}$ increases with the increase in $G r, G m, b, M$ and $t$, and it decreases $\alpha, m, \operatorname{Pr}$ and $S c$.

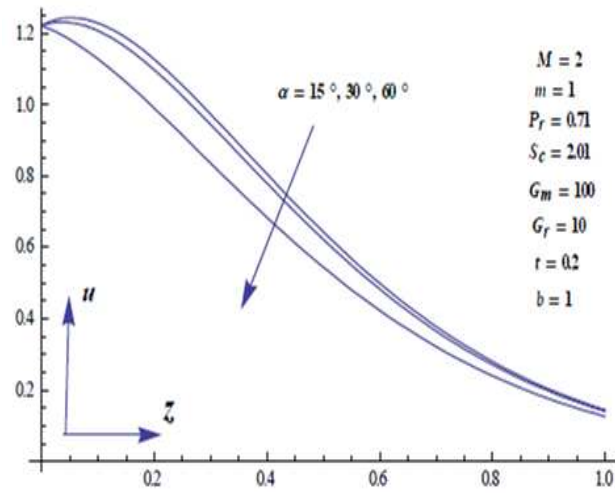

Figure 1.1: Velocity $u$ for Different Values of $\alpha$

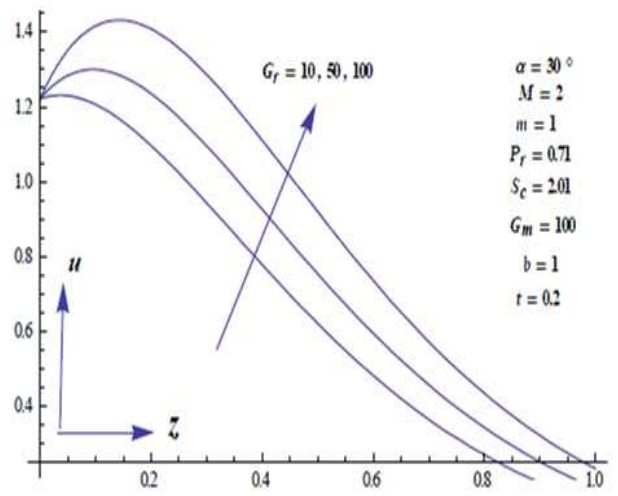

Figure 1.3: Velocity $u$ for Different Values of $G r$

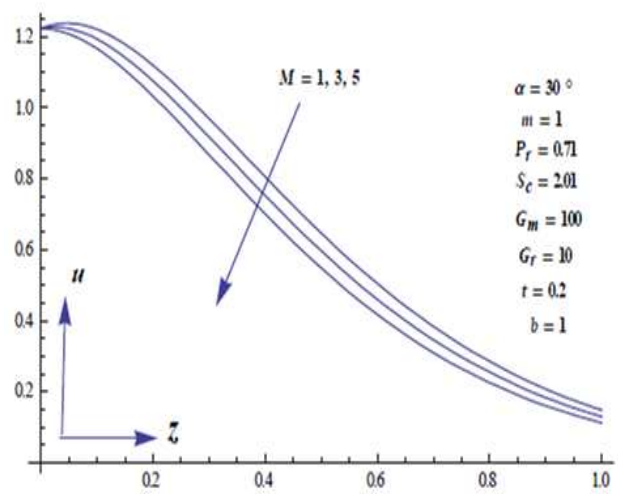

Figure 1.5: Velocity $u$ for Different Values of $M$

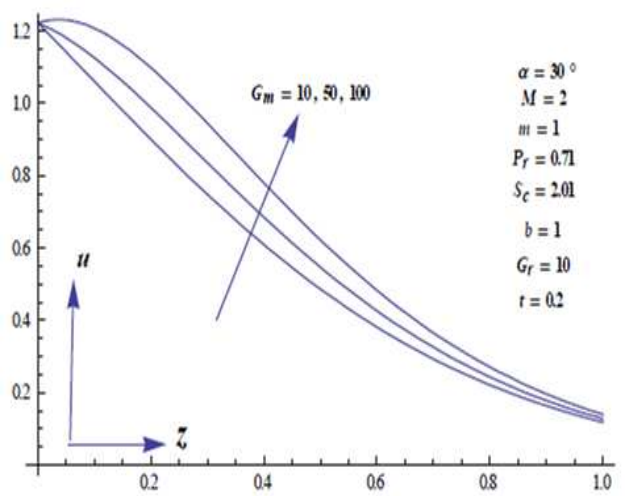

Figure 1.2: Velocity $u$ for Different Values of $G m$

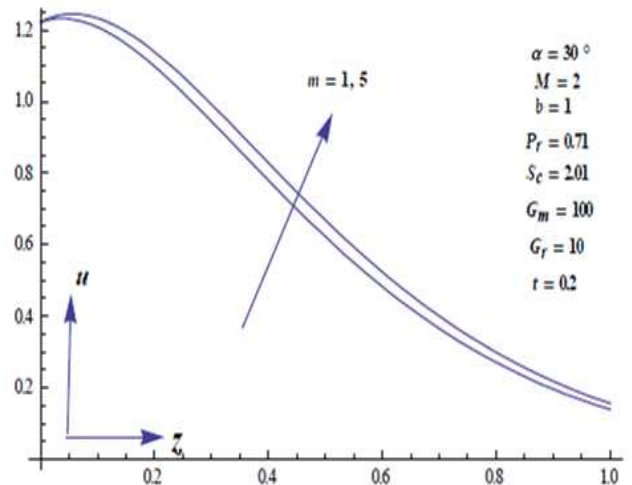

Figure 1.4: Velocity $u$ for Different Values of $m$

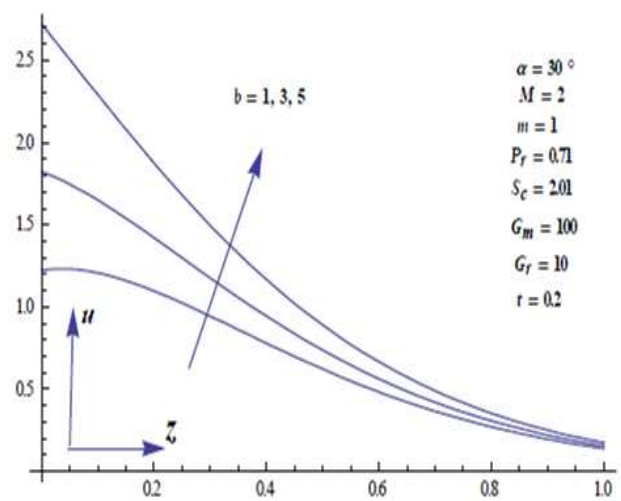

Figure 1.6: Velocity $u$ for Different Values of $b$ 


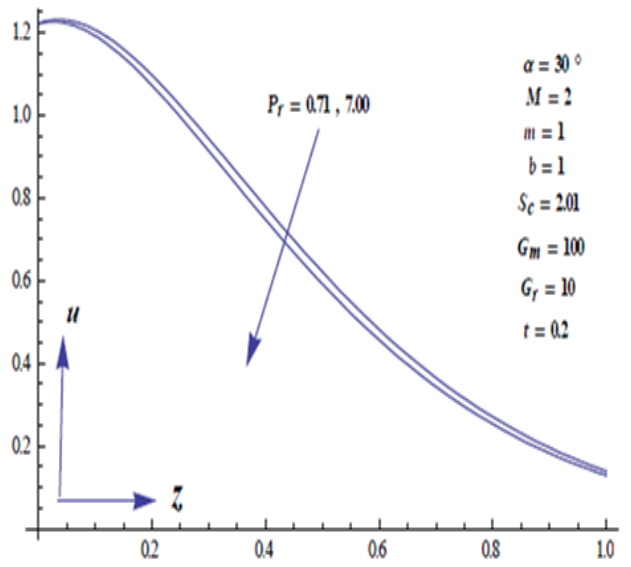

Figure 1.7: Velocity $u$ for Different Values of $\mathrm{Pr}$

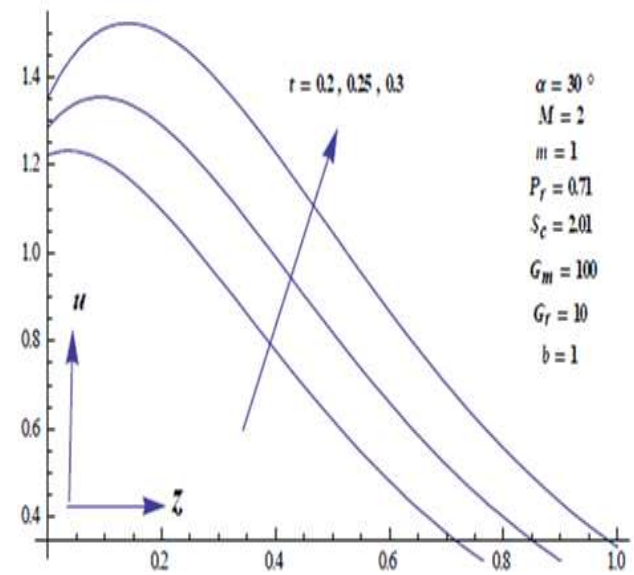

Figure 1.9 Velocity $u$ for Different Values of $t$

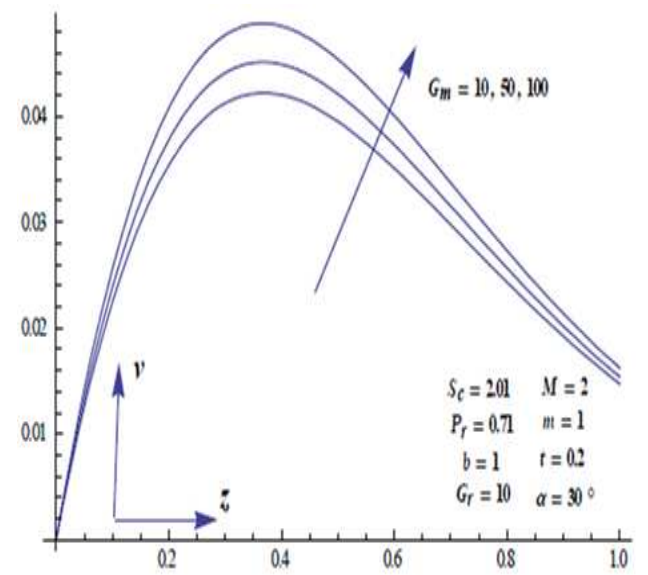

Figure 2.2: Velocity $u$ for Different Values of $G m$

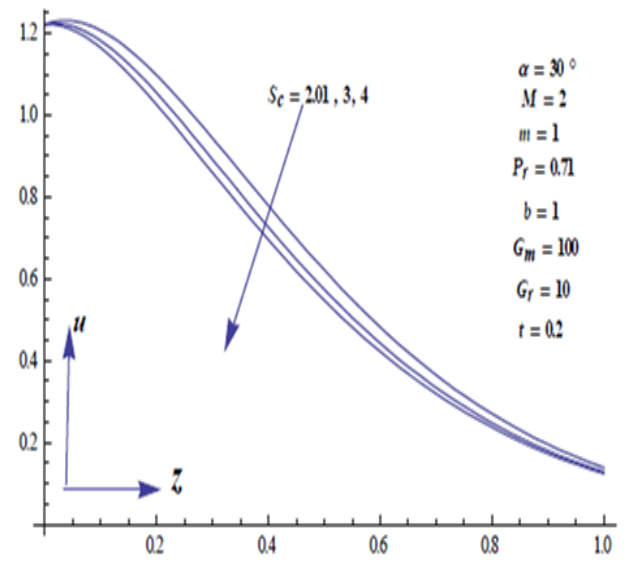

Figure 1.8: Velocity $u$ for Different Values of $S c$

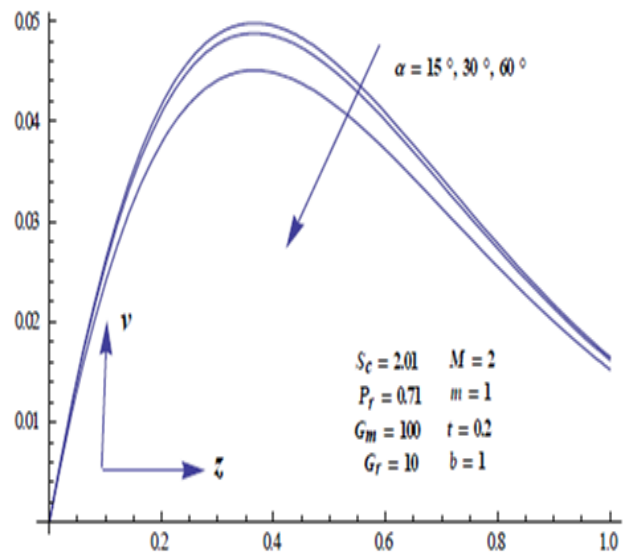

Figure 2.1: Velocity $u$ for Different Values of $\alpha$.

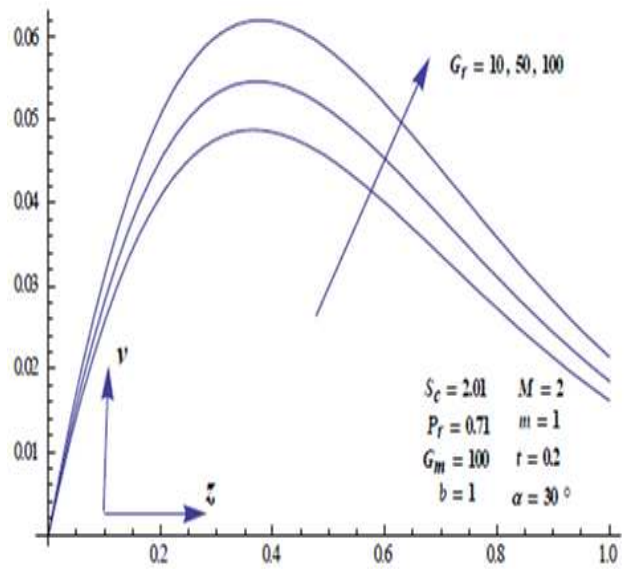

Figure 2.3: Velocity $u$ for Different Values of $G r$ 


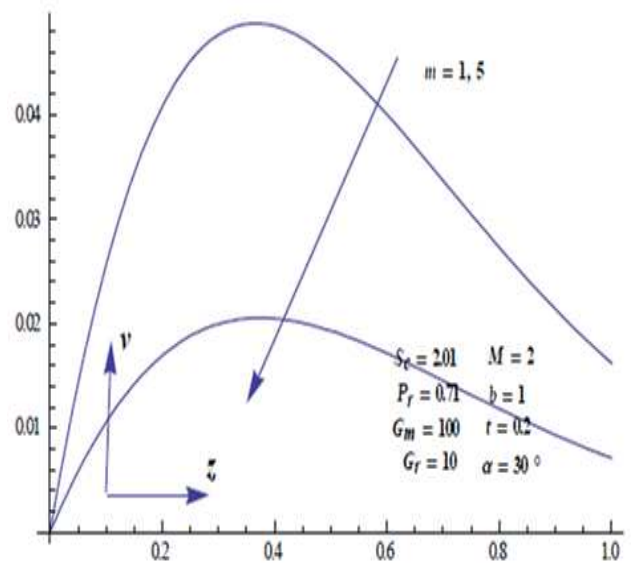

Figure 2.4: Velocity $v$ for Different Values of $m$

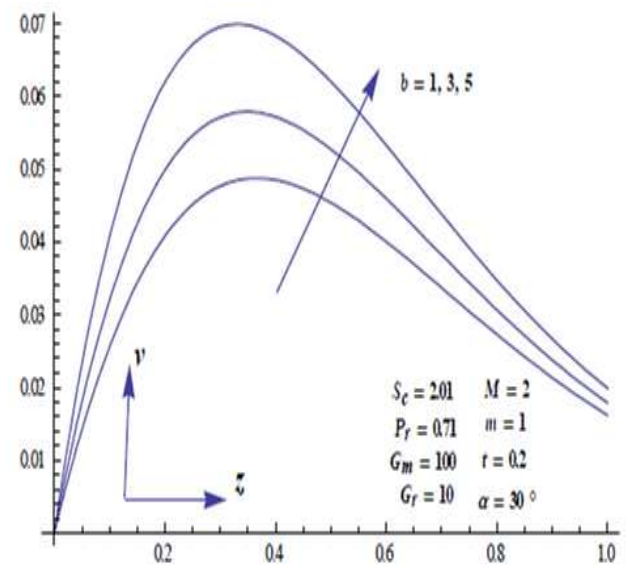

Figure 2.6: Velocity $v$ for Different Values of $b$

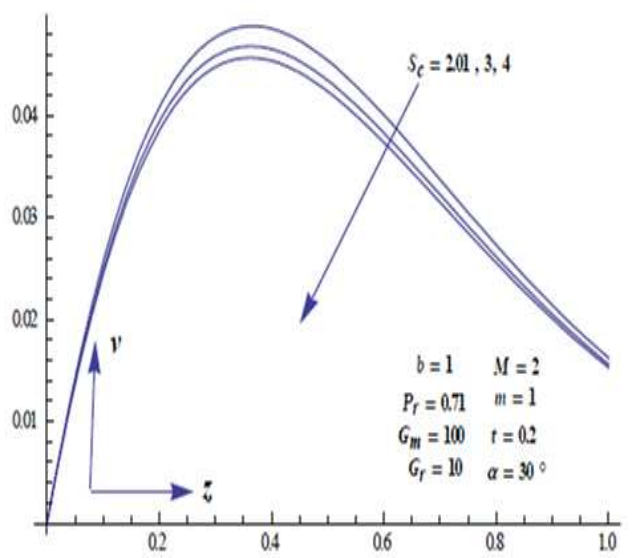

Figure 2.8: Velocity $v$ for Different Values of $S c$

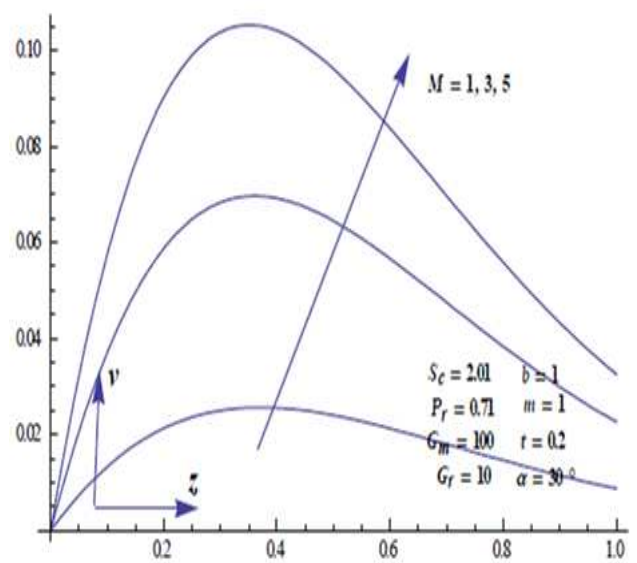

Figure 2.5: Velocity $v$ for Different Values of $M$.

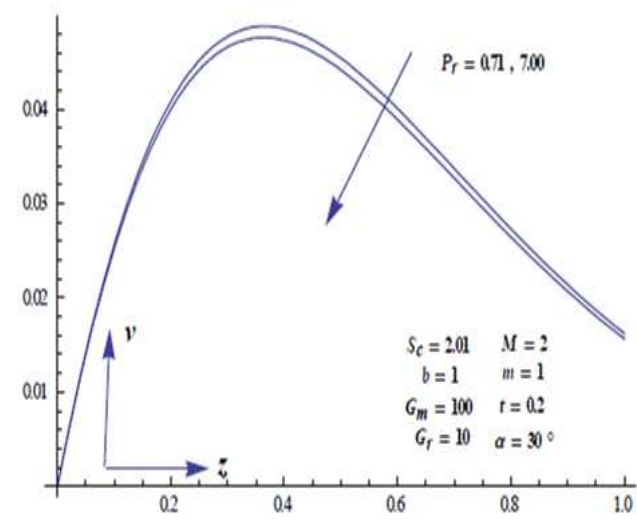

Figure 2.7: Velocity $v$ for Different Values of $\operatorname{Pr}$

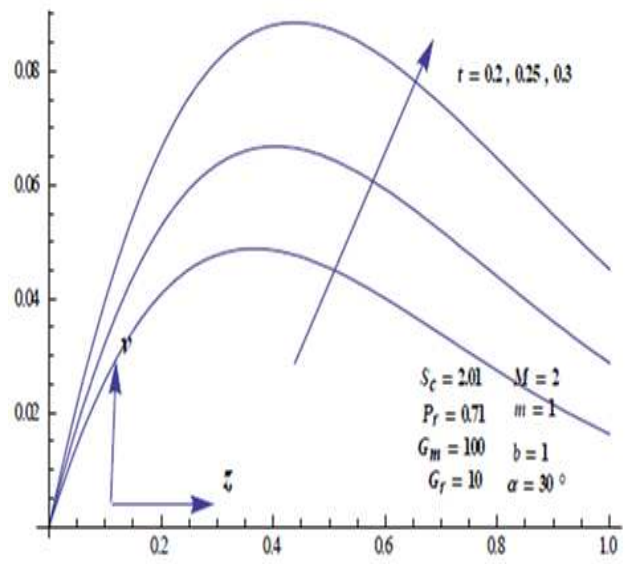

Figure 2.9: Velocity $v$ for Different Values of $t$ 
Table 1: Skin Friction for Different Parameters ( $\alpha$ in degree)

\begin{tabular}{|c|l|l|l|l|l|l|l|c|c|c|}
\hline$\alpha$ & $M$ & $m$ & $P r$ & $S c$ & $G m$ & $G r$ & $b$ & $t$ & $\tau_{x}$ & $\tau_{y}$ \\
\hline 15 & 2 & 1 & 0.71 & 2.01 & 100 & 10 & 1 & 0.2 & 0.8614 & 0.3206 \\
\hline 30 & 2 & 1 & 0.71 & 2.01 & 100 & 10 & 1 & 0.2 & 0.5522 & 0.3155 \\
\hline 60 & 2 & 1 & 0.71 & 2.01 & 100 & 10 & 1 & 0.2 & -0.5802 & 0.2968 \\
\hline 30 & 3 & 1 & 0.71 & 2.01 & 100 & 10 & 1 & 0.2 & 0.3861 & 0.4592 \\
\hline 30 & 5 & 1 & 0.71 & 2.01 & 100 & 10 & 1 & 0.2 & 0.0584 & 0.7212 \\
\hline 30 & 2 & 3 & 0.71 & 2.01 & 100 & 10 & 1 & 0.2 & 0.8167 & 0.1987 \\
\hline 30 & 2 & 5 & 0.71 & 2.01 & 100 & 10 & 1 & 0.2 & 0.8598 & 0.1284 \\
\hline 30 & 2 & 1 & 7.00 & 2.01 & 100 & 10 & 1 & 0.2 & 0.4009 & 0.3107 \\
\hline 30 & 2 & 1 & 0.71 & 3.00 & 100 & 10 & 1 & 0.2 & 0.2836 & 0.3074 \\
\hline 30 & 2 & 1 & 0.71 & 4.00 & 100 & 10 & 1 & 0.2 & 0.0984 & 0.3024 \\
\hline 30 & 2 & 1 & 0.71 & 2.01 & 010 & 10 & 1 & 0.2 & -1.5809 & 0.2815 \\
\hline 30 & 2 & 1 & 0.71 & 2.01 & 050 & 10 & 1 & 0.2 & -0.6328 & 0.2966 \\
\hline 30 & 2 & 1 & 0.71 & 2.01 & 100 & 50 & 1 & 0.2 & 1.7898 & 0.3414 \\
\hline 30 & 2 & 1 & 0.71 & 2.01 & 100 & 100 & 1 & 0.2 & 3.3366 & 0.3732 \\
\hline 30 & 2 & 1 & 0.71 & 2.01 & 100 & 10 & 3 & 0.2 & -1.2568 & 0.4056 \\
\hline 30 & 2 & 1 & 0.71 & 2.01 & 100 & 10 & 5 & 0.2 & -4.2154 & 0.5314 \\
\hline 30 & 2 & 1 & 0.71 & 2.01 & 100 & 10 & 1 & 0.3 & 2.7151 & 0.4648 \\
\hline 30 & 2 & 1 & 0.71 & 2.01 & 100 & 10 & 1 & 0.4 & 5.1524 & 0.6549 \\
\hline
\end{tabular}

\section{References}

[1] Lighthill, M. J. The response of laminar skin friction and heat transfer to fluctuations in the stream velocity. Proc. $R$. Soc. A. 1954. 224, 1-23.

[2] Katagiri M, The effect of Hall current on the magneto hydrodynamic boundary layer flow past a semi-infinite fast plate. Journal of the Physical Society of Japan. 1969. 27(4): 10511059.

[3] Pop, I. The effect of Hall currents on hydromagnetic flow near an accelerated plate. $J$. Math. Phys. Sci. 1971. 5: 375-379.

[4] Singh, A. K. and Naveen Kumar. Free convection flow past an exponentially accelerated vertical plate. Astrophysics and Space Science. 1984. 98: 245-258.

[5] Basant, K. J., Prasad, R and Rai, S. Mass transfer effects on the flow past an exponentially accelerated vertical plate with constant heat flux. Astrophysics and Space Science. 1991. 81: $125-134$.

[6] Pop, I. and Watanabe, T. Hall effects on magnetohydrodynamic boundary layer flow over a continuous moving flat plate. Acta Mech. 1995. 108: 35-47.

[7] Attia, H. A. The effect of variable properties on the unsteady Hartmann flow with heat transfer considering the Hall effect. Appl. Math. Model. 2003. 27(7): 551-563. 
[8] Attia Hazem Ali, Ahmed Mohamed Eissa Sayed. The Hall effect on unsteady MHD Couette flow with heat transfer of a Bingham fluid with suction and injection. Applied Mathematical Modelling. 2004. 28: 1027-1045.

[9] Muthucumaraswamy, R., K.E. Sathappan, Natarajan, R. Heat transfer effects on flow past an exponentially accelerated vertical plate with variable temperature. Theoret. Appl. Mech. 2008. 35(4): 323-331.

[10] Muthucumaraswamy, R., Sathappan, K. E. and Natarajan, R. Mass transfer effects on exponentially accelerated isothermal vertical plate. Int. J. of Appl. Math. and Mech. 2008. 4(6): 19-25.

[11] Rajput, U. S. and Kumar Surendra. MHD Flow past an impulsively started vertical plate with variable temperature and mass diffusion. Applied Mathematical Sciences. 2011. 5(3): $149-157$.

[12] Thamizhsudar, M. and Pandurangan, J. Combined effects of radiation and Hall current on MHD flow past an exponentially accelerated vertical plate in the presence of rotation. International Journal of Innovative Research in Computer and Communication Engineering. 2014. 2: Issue 12.

[13] Maripala Srinivas and Naikoti Kishan. Hall effects on unsteady MHD free convection flow over a stretching sheet with variable viscosity and viscous dissipation. World Applied Sciences Journal. 2015. 33(6): 1032-1041.

\section{Appendix}

$$
\begin{aligned}
& A_{1}=-1-A_{16}-e^{2 \sqrt{a} z}\left(1-A_{17}\right), A_{2}=-1+A_{16}-e^{2 \sqrt{a} z}\left(1-A_{17}\right), A_{8}=-A_{4}, \\
& A_{3}=-1+A_{20}-A_{18}\left(1-A_{21}\right), A_{4}=1+A_{23}+A_{18}\left(1-A_{24}\right) \text {, } \\
& A_{5}=-1+A_{25}-A_{19}\left(1-A_{26}\right), A_{6}=-1-A_{27}-A_{19}\left(1+A_{28}\right), A_{7}=-A_{6} \text {, } \\
& A_{9}=\frac{2 e^{-\sqrt{a} z} A_{1}}{z}(1-a t), A_{10}=\left(2 e^{\frac{-z^{2} P_{r}}{4 t}} \sqrt{t}+\sqrt{\pi} z A_{11}\right) \sqrt{P_{r}}, A_{11}=-1+\operatorname{erf}\left[\frac{z \sqrt{P_{r}}}{2 \sqrt{t}}\right] \text {, } \\
& A_{12}=-1+\operatorname{erf}\left[\frac{z \sqrt{S_{c}}}{2 \sqrt{t}}\right], A_{13}=e^{\frac{a t}{-1+P_{r}}-z \sqrt{\frac{a P_{r}}{-1+P_{r}}}}, A_{14}=e^{\frac{a t}{-1+S_{c}}-z \sqrt{\frac{a S_{c}}{-1+S_{c}}}}, \\
& A_{15}=1+A_{29}+e^{2 \sqrt{a+b} z} A_{30}, A_{16}=\operatorname{erf}\left[\frac{2 \sqrt{a} t-z}{2 \sqrt{t}}\right], A_{17}=\operatorname{erf}\left[\frac{2 \sqrt{a} t+z}{2 \sqrt{t}}\right] \text {, } \\
& A_{18}=e^{-2 z \sqrt{\frac{a P_{r}}{-1+P_{r}}}}, A_{19}=e^{-2 z \sqrt{\frac{a S_{c}}{-1+S_{c}}}}, A_{20}=\operatorname{erf}\left[\frac{z-2 t \sqrt{\frac{a P_{r}}{-1+P_{r}}}}{2 t}\right] \text {, } \\
& A_{21}=\operatorname{erf}\left[\frac{z+2 t \sqrt{\frac{a P_{r}}{-1+P_{r}}}}{2 t}\right], A_{22}=\operatorname{erf}\left[\frac{2 t \sqrt{\frac{a}{-1+P_{r}}}-z \sqrt{P_{r}}}{2 t}\right] \text {, } \\
& A_{23}=\operatorname{erf}\left[\frac{2 t \sqrt{\frac{a}{-1+P_{r}}}-z \sqrt{P_{r}}}{2 t}\right], A_{24}=\operatorname{erf}\left[\frac{2 t \sqrt{\frac{a}{-1+P_{r}}}+z \sqrt{P_{r}}}{2 t}\right] \text {, } \\
& A_{25}=\operatorname{erf}\left[\frac{z-2 t \sqrt{\frac{a S_{c}}{-1+S_{c}}}}{2 t}\right], A_{26}=\operatorname{erf}\left[\frac{z+2 t \sqrt{\frac{a S_{c}}{-1+S_{c}}}}{2 t}\right] \text {, }
\end{aligned}
$$




$$
\begin{aligned}
& A_{27}=\operatorname{erf}\left[\frac{2 t \sqrt{\frac{a}{-1+S_{c}}}-2 \sqrt{S_{c}}}{2 t}\right], A_{28}=\operatorname{erf}\left[\frac{2 t \sqrt{\frac{a}{-1+S_{c}}}+2 \sqrt{S_{c}}}{2 t}\right], \\
& A_{29}=\operatorname{erf}\left[\frac{2 \sqrt{a+b} t-z}{2 \sqrt{t}}\right], A_{30}=\operatorname{erf}\left[\frac{2 \sqrt{a+b} t+z}{2 \sqrt{t}}\right], a=\frac{M(1-i m)}{1+m^{2}} .
\end{aligned}
$$

\section{List of Symbols}

$u \quad$ The primary velocity of the fluid

$v \quad$ The secondary velocity of the fluid

$g \quad$ The acceleration due to gravity

$t \quad$ Time

$m \quad$ Hall current parameter

$T \quad$ Temperature of the fluid

$C \quad$ Species concentration in the fluid

$b \quad$ Acceleration parameter

D Mass diffusion coefficient

$T_{\mathrm{w}} \quad$ Temperature of the plate at $\mathrm{z}=0$

$C_{\mathrm{w}} \quad$ Species concentration at the plate $\mathrm{z}=0$

$B_{0} \quad$ Magnetic field

$K \quad$ Thermal conductivity of the fluid

$G_{\mathrm{r}} \quad$ Thermal Grashof number

$G_{\mathrm{m}} \quad$ Mass Grashof number

$P_{\mathrm{r}} \quad$ Prandtl number

$S_{\text {c }} \quad$ Schmidt number

$M \quad$ The magnetic parameter

$C_{\mathrm{p}} \quad$ Specific heat at constant pressure

$\bar{u} \quad$ Dimensionless Primary velocity

$\bar{v} \quad$ Dimensionless secondary velocity

$\bar{b} \quad$ Dimensionless acceleration parameter

$\bar{t} \quad$ Dimensionless time

$\bar{C} \quad$ Dimensionless concentration

$\alpha \quad$ Angle of inclination of plate

$\beta \quad$ Volumetric coefficient of thermal expansion

$\beta^{*} \quad$ Volumetric coefficient of concentration expansion

$\nu \quad$ Kinematic viscosity

$\rho \quad$ Density

$\mu \quad$ Coefficient of viscosity

$\sigma \quad$ Electrical conductivity

$\omega_{\mathrm{e}} \quad$ Cyclotron frequency of electrons

$\tau_{e} \quad$ Electron collision time

$\theta \quad$ Dimensionless temperature 